Pure \& Appl. Chem., Vol. 57, No. 1, pp. 89-103, 1985.

Printed in Great Britain.

(C) 1985 IUPAC

\title{
Phase equilibria in fluid mixtures
}

\author{
Maxwell L. McGlashan \\ Department of Chemistry, University College London, \\ 20 Gordon Street, London WC1H 0AJ, U.K.
}

(The fifth Rossini Lecture given at the IUPAC Conference on Chemical Thermodynamics held at McMaster University, Hamilton, Ontario, Canada on 14 August 1984)

\begin{abstract}
Phase diagrams for binary fluid mixtures are reviewed with respect to experimental methods used for their study; the kinds of phase diagram obtained in practice, especially those including one or more of azeotropy, the coexistence of three fluid phases, and the coexistence of two fluid phases ("gas-gas immiscibility") at temperatures above the critical temperature of either pure component; and the extent to which modern theoretical treatments can be used to predict what actually happens at different pressures, temperatures, and compositions.
\end{abstract}

\section{INTRODUCTION}

It is well known that, at pressures close to atmospheric, binary fluid mixtures lead to various kinds of phase diagram such as those shown in Fig. 1, where temperature $T$ is plotted against mole fraction $x$ at
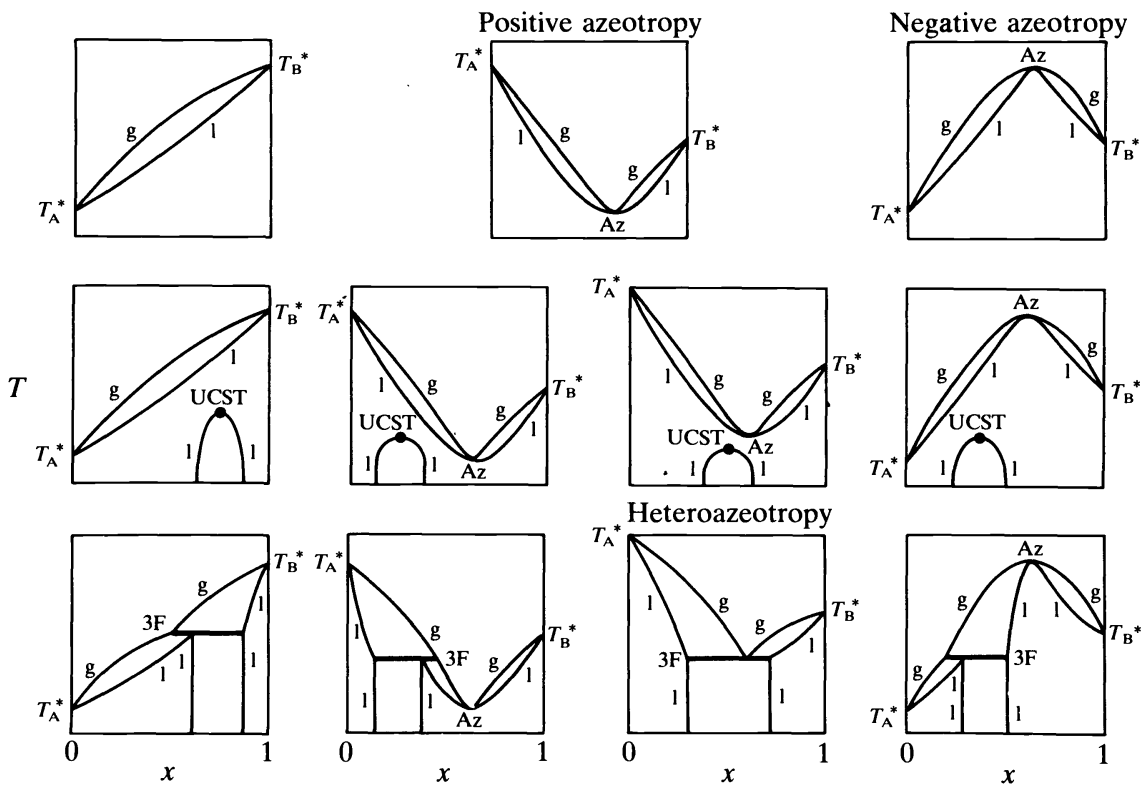

Fig. 1. Boiling-temperature diagrams at pressures near atmospheric.

constant (atmospheric) pressure, and where in the top row ordinary (liquid + vapour) equilibrium is shown with an example each of positive and negative azeotropy; where in the middle row a (liquid + liquid) equilibrium terminating in an upper critical solution temperature (UCST) intrudes; and 
where in the bottom row the three-fluid-phase consequences of the intersection of the two two-phase regions are shown. Positive azeotropy can lead either to three fluid phases ("3F") in which the sequence of phases from left to right is liquid, liquid, and vapour; or to "heteroazeotropy": namely, the occurrence of three fluid phases in which the sequence is liquid, vapour, and liquid, the three fluid phases then coexisting at a lower temperature than the boiling temperature of either pure component. Negative azeotropy is not shown as leading to heteroazeotropy; that would imply the coexistence of four fluid phases, and although that state of affairs is not forbidden by the phase rule no evidence for the occurrence of four coexisting fluid phases has ever been found for a binary mixture. (Why are we so ready to assume that four fluid phases can never coexist in a binary mixture? Four phases including at least one solid phase can of course coexist at a quadruple point on a binary phase diagram.)

Already, even for pressures close to atmospheric, we have introduced more than is commonly found in any textbook of physical chemistry. But there is no textbook as far as I am aware in which anything whatever is said about such diagrams at higher pressures and especially in the critical region: at pressures of about $4 \mathrm{MPa}$ or 40 times atmospheric pressure. (Why do nearly all fluids have critical pressures around $4 \mathrm{MPa}$, so that the quotient $V_{\mathrm{m}}^{\mathrm{c}} / T \mathrm{c}$ of the critical molar volume $V_{m}^{c}$ and the critical temperature $T$ is always around $0.6 \mathrm{~cm}^{3} \cdot \mathrm{mol}^{-1} \cdot \mathrm{K}^{-1}$ ?)

This neglect in the education of chemists of an important part of physical chemistry is most deplorable, and indeed almost unbelievable though consistent with physical chemists' traditional neglect of pressure as a variable, and explicable if not excusable in terms of each textbook writer having taken over the bounds of his subject matter from his predecessors. Quite apart from the intrinsically interesting variety of behaviour that can be found in it and the considerable challenge it offers to the experimentalist, the field is extremely and increasingly important in contemporary high-pressure industrial processes such as the transmission in pipelines of natural gas (impure methane) and those designed to produce synthetic methane from coal, or petrol or methanol from methane; in highpressure extractions (for example in the decaffeination of coffee beans with carbon dioxide); in the phase-transfer techniques, often at high pressures, that are achieving increasing prominence in synthetic chemistry; in "supercritical fluid chromatrography" (Ref. 1); and to geologists in their studies of the processes, usually at very high temperatures and pressures, that have resulted in the earth's crust as we now find it.

In this lecture I shall confine myself to binary fluid mixtures with pressure $p$, temperature $T$, and mole fraction $x$ as variables. I shall not deal at all with the molar volumes $V_{m}$ of the phases, with the separation of solid phases, or with ternary let alone more complex mixtures; each of these would have added greatly to the interest, but too much to the length, of the lecture. For fuller accounts of the field the reader should turn to the literature (Refs. 1-7).

\section{EXPERIMENTAL METHODS}

Two quite different plans of measurement are available (Fig. 2):
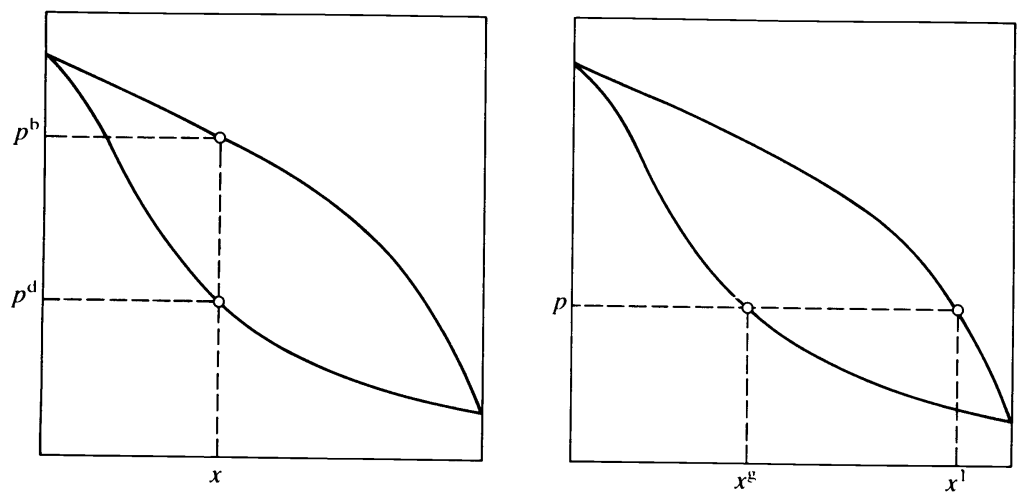

Fig. 2. Plot of vapour pressure $p$ against mole fraction $x$ to illustrate two distinct plans for experimental measurements. 
(i) measurements of the dew pressure $p^{\mathrm{d}}$ and the bubble pressure $p^{\mathrm{b}}$ of a mixture whose composition $x$ is known by quantitative synthesis, and (ii) measurements of the pressure $p$ and the compositions $x g$ and $x^{1}$ of coexisting phases by taking samples and analysing them for example by g.1.c. or by measurements of thermal conductivity.

The first plan has been extensively used for example by our group (8-10), our newer apparatus being shown in Fig. 3, but has the disadvantages: in effect

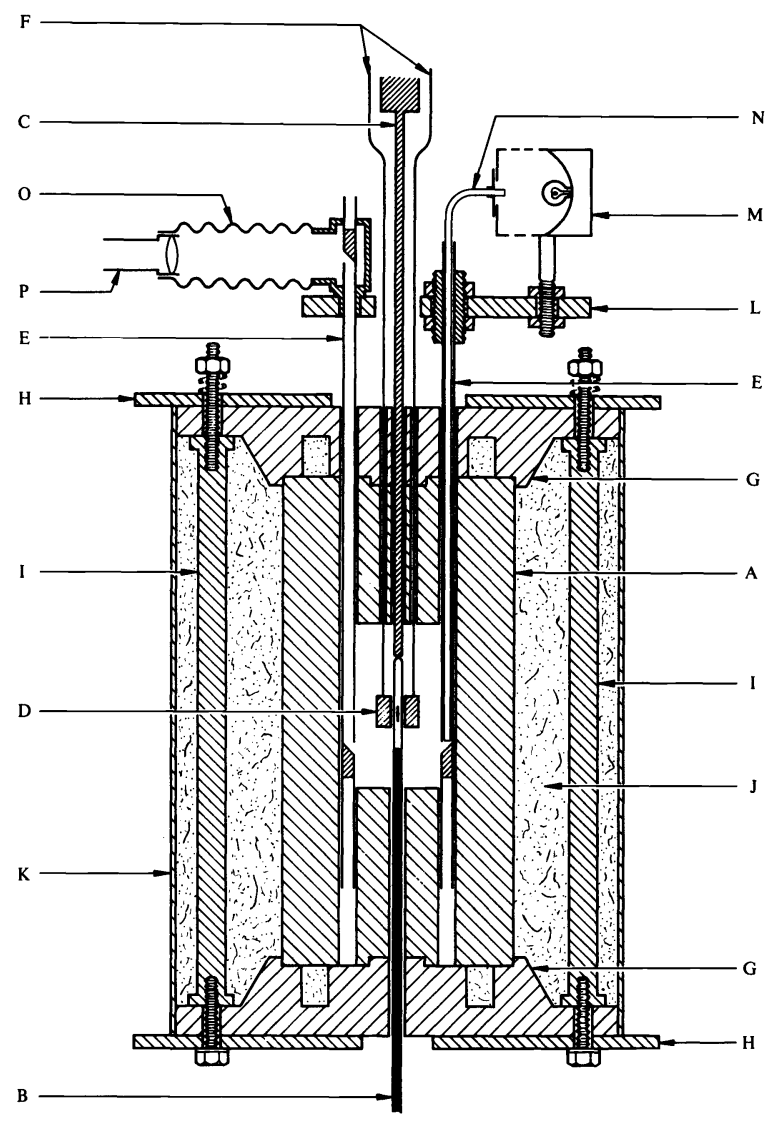

Fig. 3. Hugill and McGlashan's experimental arrangements for measurements of dew pressure $p^{\mathrm{d}}$ and bubble pressure $p^{\mathrm{b}}$ for a synthetic mixture of mole fraction $x$.

of placing too much weight on too few synthetic mixtures (because of the tedium of making them, and keeping them, sufficiently accurately and sufficiently air-free); of needing many hours to reach equilibrium after any adjustment; of its being awkward and often impossible, except at densities close to the critical, to arrange suitable volumes for a given sample at both the dew pressure and the bubble pressure; and of the mixture being in contact with mercury. In the apparatus shown in Fig. 3 we avoided spoiling the temperature control from the aluminium-alloy-block thermostat just where it is most important by using a double periscope to light and to observe the mercury-to-fluid and the fluid-to-fluid menisci and so to avoid a window.

The second plan can be further divided into static methods in which the two phases are, eventually, brought to equilibrium; and dynamic or recirculatory methods in which the less dense phase is pumped from the top of the cell round and back into the bottom of the cell and through the more dense phase. An example of a static cell is shown in Fig. 4 (Ref. 11); it is designed for analysis of the two phases, after reduction of their 


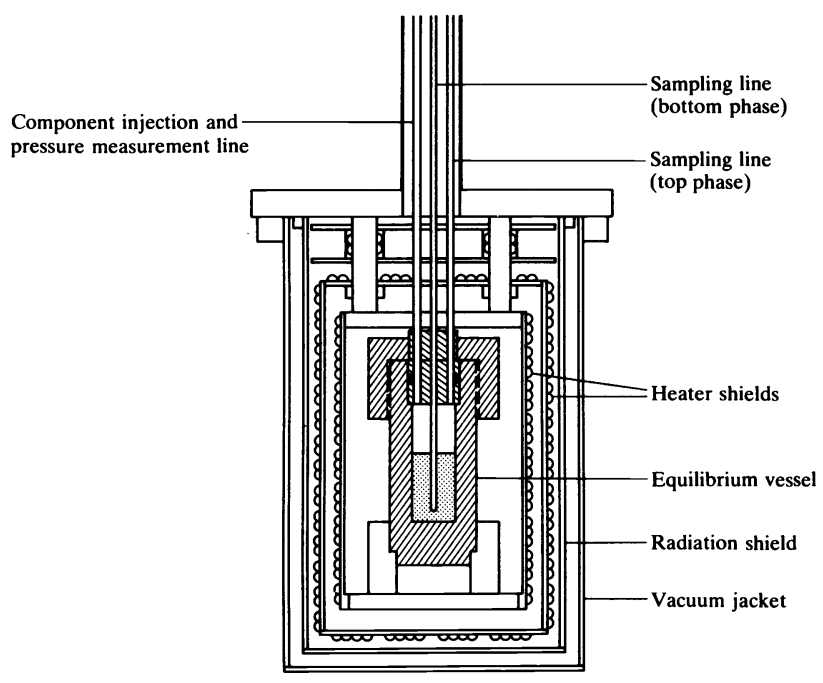

Fig. 4. A cell suitable for static measurements of the mole fractions $x^{g}$ and $x^{1}$ of coexisting mixtures at the pressure $p$.

pressures, with a gas-liquid chromatograph. The chief modern exponents of the dynamic method are Streett and his colleagues, a version of whose apparatus (Ref. 12) is shown in Fig. 5 .

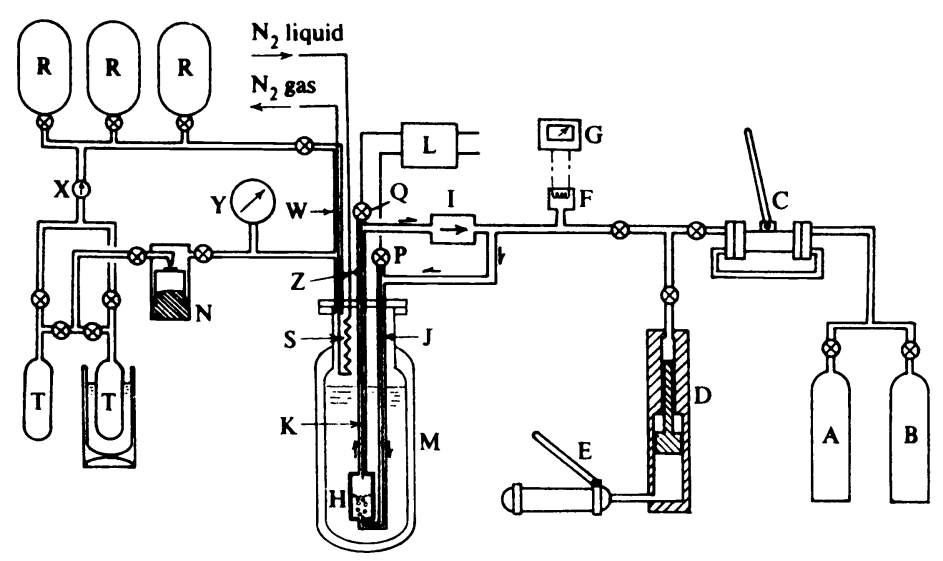

Fig. 5. Streett and Calado's apparatus. A and B, cylinders containing the gases to be studied; C, two-stage diaphragm compressor; D, pressure intensifier; E, hydraulic pump; F, manganin pressure gauge; G, Wheatstone bridge; H, pressure vessel; I, magnetically operated pump; $J$ and $K$, sampling lines; $L$, thermal-conductivity gas analyser; $M$, cryostat; $N$, Cartesian manostat; $P$ and $Q$, sampling valves; $R$, gas cylinders for storing refrigerant; $\mathrm{S}$, coil of copper tubing ( $6.35 \mathrm{~mm}$ o.d., $5.0 \mathrm{~mm}$ i.d.); T, stainless-steel cylinders; W, counterflow heat exchanger; $X$, check valve; $Z$, thermocouple junction.

Experimental methods for the study of the phase equilibria of fluid mixtures have been extensively reviewed by Schneider (13) and by Young (14). 


\section{THE SIMPLEST KIND OF PHASE DIAGRAM}

For even the simplest kind of binary phase diagram, without any of the complications of azeotropy or the coexistence of three fluid phases or gasgas immiscibility, it is difficult enough to present three variables in twodimensional diagrams. Even if we slowly become familiar in $(p, T, x)$ space with the three two-dimensional projections and the three kinds of twodimensional sections we must still master those for $\left(V_{m}, T, x\right)$ and for the other two sets, as well as those including other variables. Here in Fig. 6
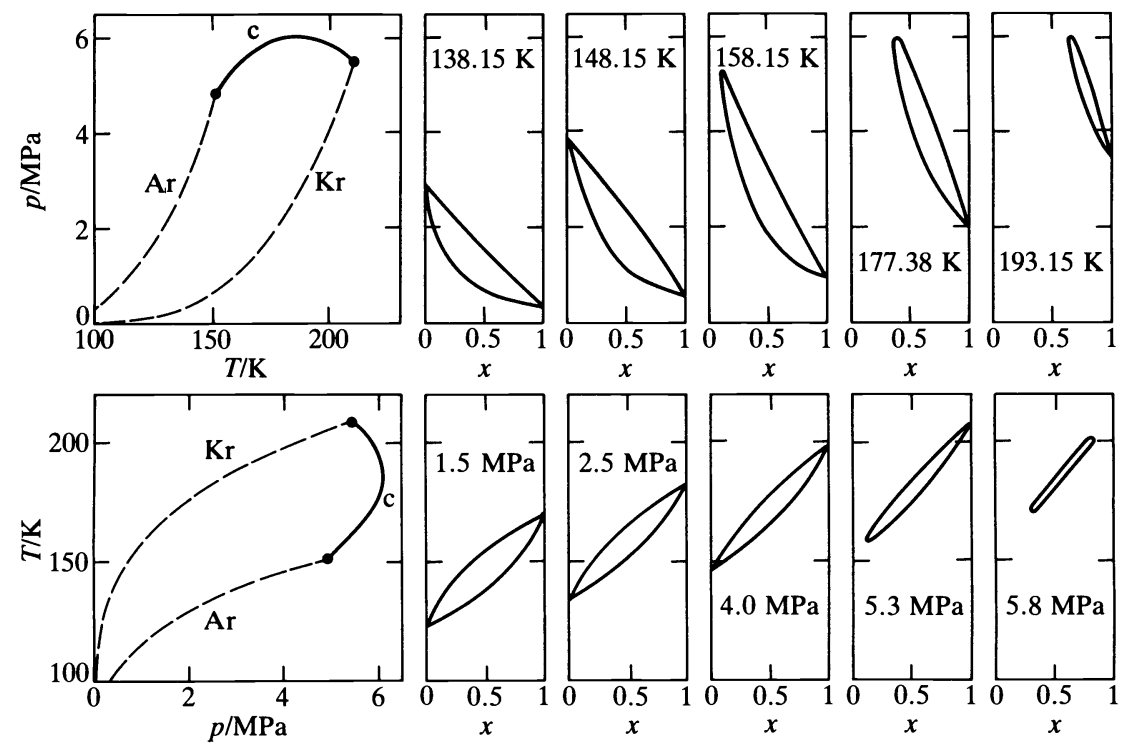

Fig. 6. The $p(T)$ projection and five $p(x)$ sections in the upper part, and the $T(p)$ projection and five $T(x)$ sections in the lower part, for $\{(1-x) \mathrm{Ar}+x \mathrm{Kr}\}$.

is one method of presentation for $\{(1-x) \mathrm{Ar}+x \mathrm{Kr}\}$ studied at the van der Waals Laboratory in Amsterdam (Ref. 15), combining a $p(T)$ projection with corresponding isothermal sections, and the $T(p)$ projection with isobaric sections. Even in this simplest kind of diagram (we shall for the most part keep in the following diagrams to the convention: --- for pure substances; .... for azeotropes; — for critical curves; - for the coexistence of two fluid phases; and -.- for the coexistence of three fluid phases) we see that it is not always enough to transform from $p(x)$ sections to $T(x)$ sections by rotation through $\pi$ and reflexion.

Does it help to show, as in Fig. 7, the diagram drawn as though in three

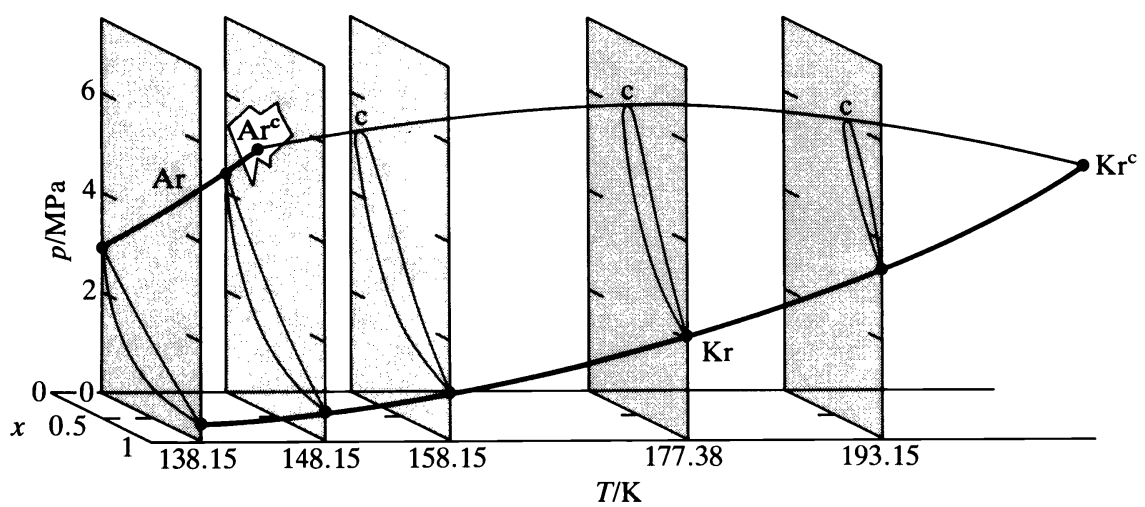

Fig. 7. A "three-dimensional" version of Fig. 6. 
dimensions? The answer to that question will depend, apart from questions about the skill of the draftsman, on the extent to which an individual is capable of seeing such drawings as though they were three-dimensional models. Can you, for example, from Fig. 7, easily draw say the $T(x)$ projection or even some $T(x)$ sections?

\section{RETROGRADE CONDENSATION}

Retrograde condensation occurs when the dew-pressure curve of an isothermal section (or the bubble-temperature of an isobaric section) is intersected twice by a line of constant composition. As an example, we show in Fig. 8 part of the isothermal section at $177.38 \mathrm{~K}$ from Fig. 6 . The curve marked g

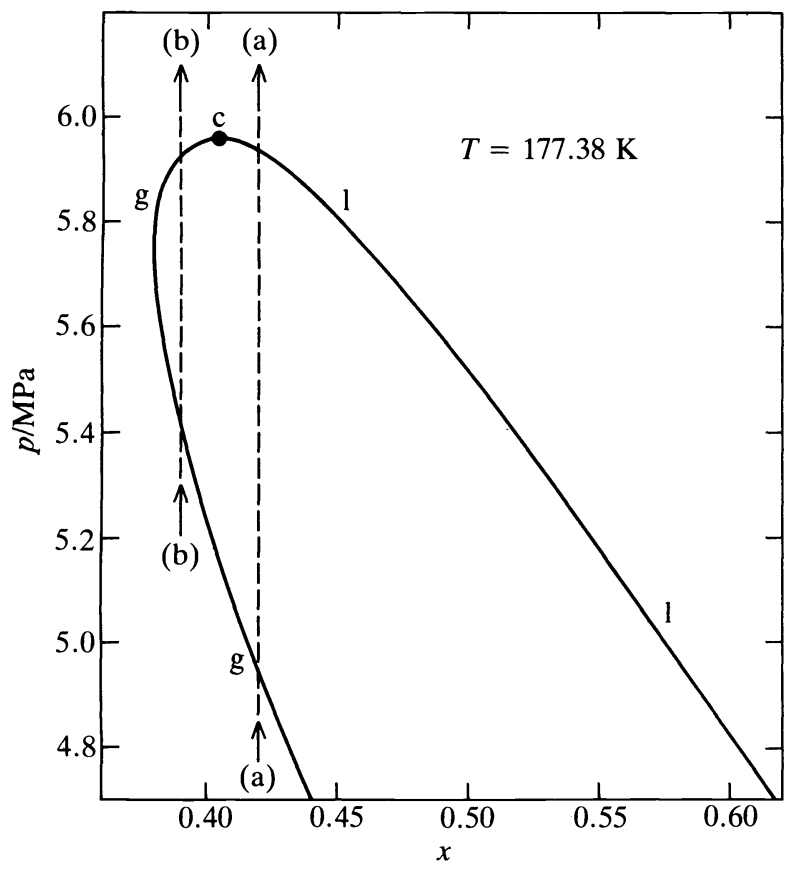

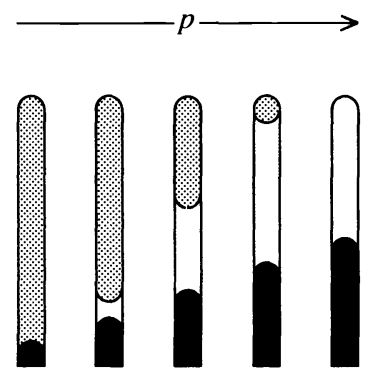

(a)

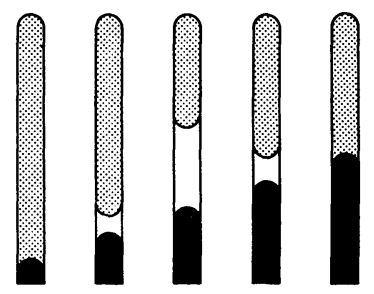

(b)

Fig. 8. Retrograde condensation.

is the dew-pressure curve and that marked 1 is the bubble-pressure curve, the two being separated at the critical point $c$. When the gaseous mixture (a) is compressed it condenses in the ordinary way as shown diagrammatically on the upper right-hand side. When the gaseous mixture (b) is compressed, however, it begins to condense to liquid but as the compression is continued the liquid phase at first grows but then shrinks and finally disappears, as shown diagrammatically on the lower right-hand side.

It is obvious that there will be pathways of retrograde condensation for any fluid mixture. It is easy to understand the bewilderment that must have greeted the first observation of this phenomenon. A gaseous mixture was expected to condense to a liquid when the pressure was increased at constant temperature (or when the temperature was decreased at constant pressure). Moreover, at first sight the (most entertaining!) series of observations labelled (b) on the lower right-hand side of Fig. 8 appears to the experimentalist to defy the rule that at given temperature an increase of pressure must result in a decrease of volume.

\section{AZEOTROPY}

An azeotrope differs from a critical mixture; for an azeotrope $x^{\alpha}=x^{\beta}$ but $V_{\mathrm{m}}^{\alpha} \neq V_{\mathrm{m}}^{\beta}$, while for a critical mixture $x^{\alpha}=x^{\beta}$ and $V_{\mathrm{m}}^{\alpha}=V_{\mathrm{m}}^{\beta}$. Azeotropy may, but need not, persist up to the critical line. According to Wichterle (7) azeotropy can be classified as in Fig. 9; in cases (a) and (c) it persists up to the critical line but in (b) and the Bancroftian (d) it does not. 

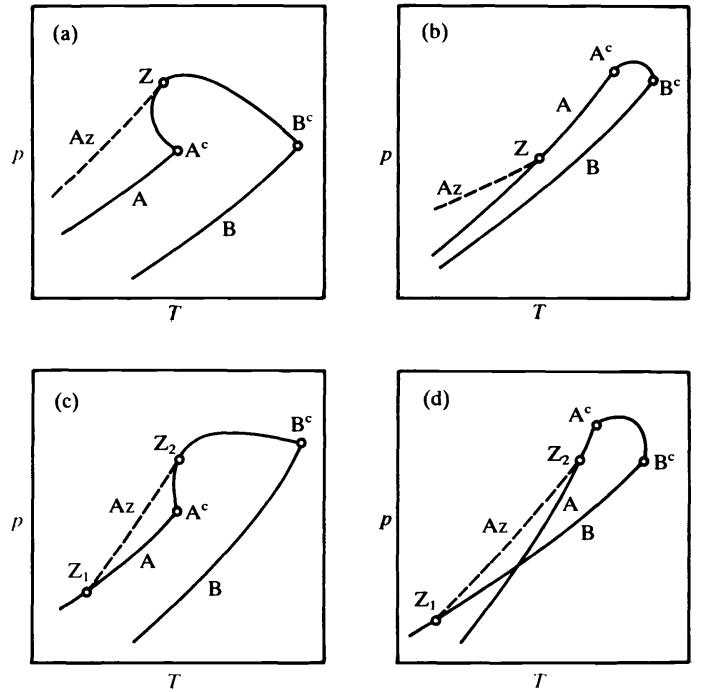

Fig. 9. Wichterle's classification of azeotropes.

Like most attempts at classification of such phase diagrams, however, Fig. 9 is, as we shall see, incomplete. In the meantime we show in Fig. 10 for $\left\{(1-x) c-\mathrm{C}_{6} \mathrm{H}_{12}+\mathrm{C}_{6} \mathrm{~F}_{6}\right\}$ (Ref. 16) an example of a positive azeotrope of

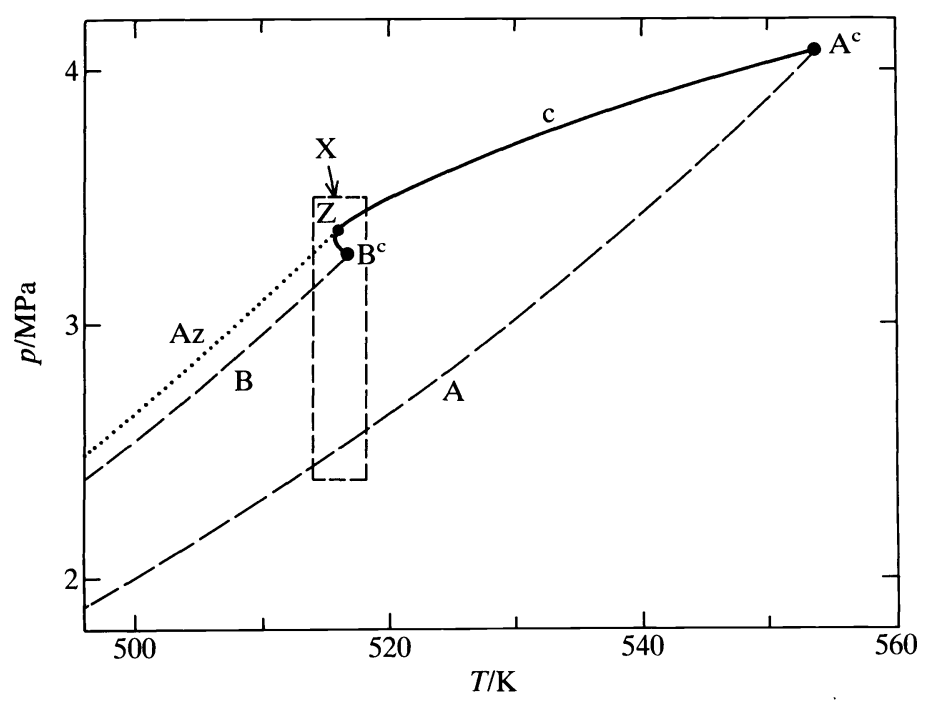

Fig. 10. The $p(T)$ projection for $\left\{(1-x) c-\mathrm{C}_{6} \mathrm{H}_{12}+x \mathrm{C}_{6} \mathrm{~F}_{6}\right\}$
as an example of positive azeotropy.

type (a) persisting up to the critical line. A positive azeotrope has a maximum in the curve of $p(x)$ at given $T$; the inverse behaviour is called negative azeotropy but is relatively uncommon. The region around the point $\mathrm{Z}$ of intersection of the azeotropic line $\mathrm{Az}$ and the critical line $\mathrm{c}$ is drawn on a larger scale in Fig. 11. We see for example two critical pressures at temperatures $T_{2}, T_{3}$, and $T_{4}$, and at $T_{3}$ for the isotherm passing through $\mathrm{Z}$ that the $p(x)$ section meets the critical line at a horizontal cusp. 

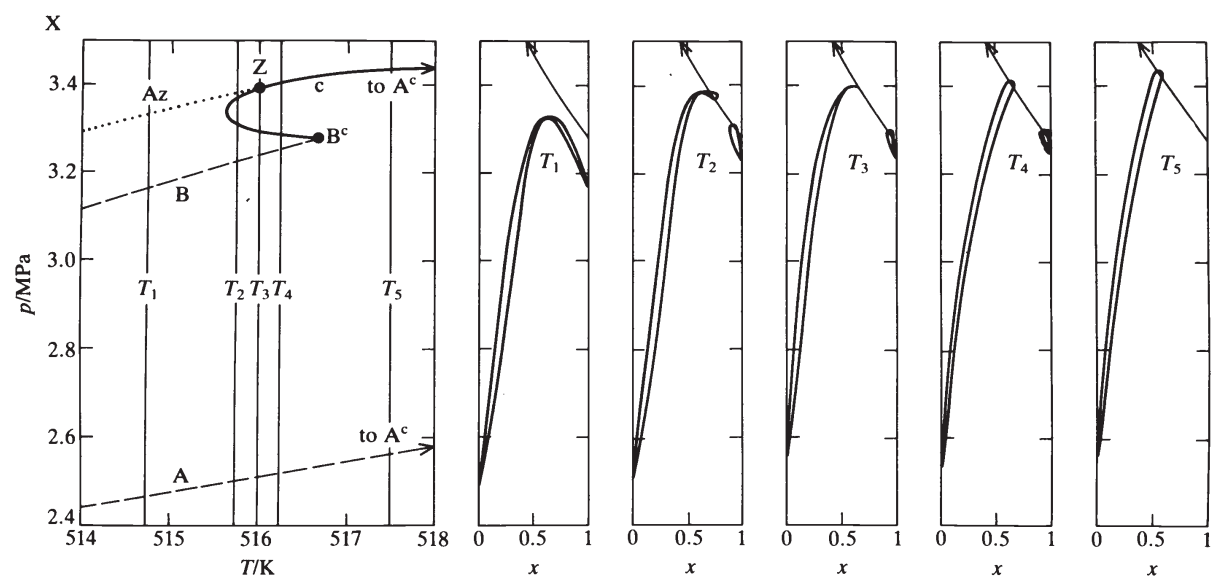

Fig. 11. The $p(T)$ projection and five $p(x)$ sections for the small part of Fig. 10 marked "X". In the $p(x)$ sections the separateness of the dew and bubble curves has been a little exaggerated for the sake of clarity.

In Fig. 12 a $(p, T, x)$ diagram is drawn for $\left\{(1-x) \mathrm{C}_{6} \mathrm{H}_{6}+x_{6} \mathrm{~F}_{6}\right\}$ for which Gaw and Swinton (17) had found a double azeotrope at temperatures near room

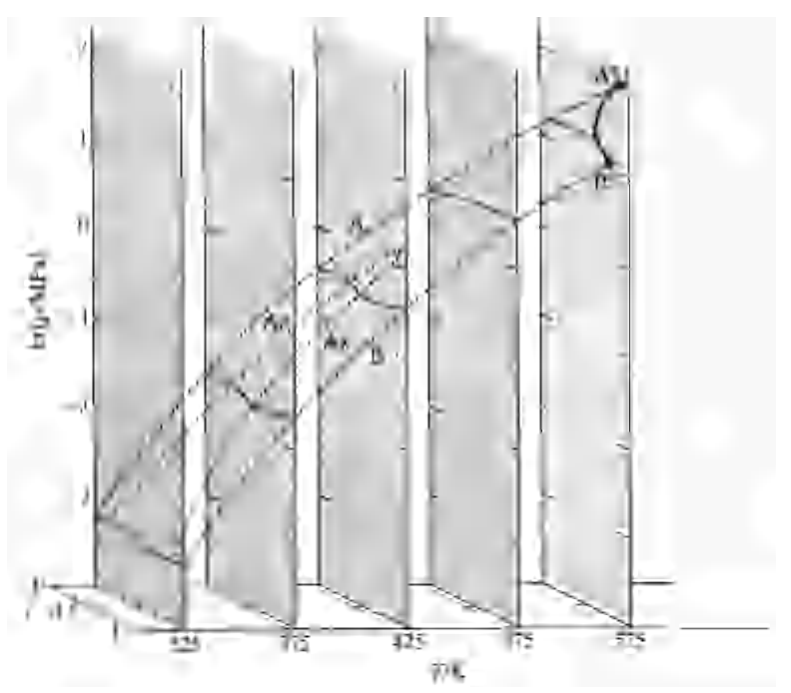

Fig. 12. A "three-dimensional" diagram showing double azeotropy in $\left\{(1-x) \mathrm{C}_{6} \mathrm{H}_{6}+\mathrm{x}_{6} \mathrm{~F}_{6}\right\}$.

temperature: a positive azeotrope in benzene-rich mixtures and a negative one in hexafluorobenzene-rich mixtures. Figure 12 shows (Refs. 19, 20) how the double azeotrope disappears at a mole fraction near the middle of the range at a point at which $(\partial p / \partial x)_{T}=0$ and $\left(\partial^{2} p / \partial x^{2}\right)_{T}=0$, that is to say at a horizontal point of inflexion of $p(x)$. That it needs a little imagination to see the maximum and minimum in $p(x)$ at each of the three temperatures for which it has been drawn illustrates the difficulties that arise from scale in drawing such diagrams. When each isothermal $p(x)$ curve is drawn (Refs. 18, 19) on a much larger scale, the maximum and minimum are clear, as are the separations between dew pressure and bubble pressure at given composition, any inference drawn by extrapolation in the first paper (Ref. 18) having been confirmed experimentally in the second (Ref. 19). 


\section{COEXISTENCE OF THREE FLUID PHASES}

Of the many binary mixtures for which three fluid phases ("3F") are known to coexist, there are, even now, very few for which the phase equilibria have been studied over the whole of the relevant range of $p, T$, and $x$. Only extremely rarely has such a study been completed in a single apparatus; different parts of the phase diagram have been studied in different laboratories, but then they have usually been studied for different pairs of substances! It is not going too far to assert that there is no binary mixture having a $3 F$ range for which a complete phase diagram can be drawn, at least without a good deal of guesswork. Even for the very few for which most of the diagram can be pieced together from different studies, the presentation is beset by problems of scale, problems of the kind already illustrated by our need to redraw a small part of Fig. 10 on a larger scale in Fig. 11, and within Fig. 11 by our need sometimes to exaggerate the differences between the compositions of pairs of coexisting phases so as to be able to see them separately. The fullest modern compilation (Ref. 20) contains no example of a reasonably complete phase diagram including a $3 \mathrm{~F}$ line. Indeed, one might have wondered whether such behaviour were exceptional, if one were not aware that three fluid phases coexist in a wide variety of common industrially important mixtures like $\left\{(1-x) \mathrm{CO}_{2}+x \mathrm{C}_{n} \mathrm{H}_{2 n}+2\right\}$ with $n \geqslant 8,\left\{(1-x) \mathrm{CH}_{4}+x \mathrm{C}_{n} \mathrm{H}_{2 n+2}\right\}$ with $n \geqslant 6$, and $\left\{(1-x) \mathrm{H}_{2} \mathrm{O}+x \mathrm{C}_{n} \mathrm{H}_{2 n+2}\right\}$. behaviour like that in Fig. 6, including some examples of azeotropy like that in Fig. 10, Ref. 20 contains a quite substantial minority of examples of "gas-gas immiscibility" at temperatures around the critical temperature of the less volatile component, implying in each such case an as yet unstudied 3F line terminating in an upper critical end point ("UCEP") close to the critical temperature of the more volatile component. An example familiar to us, and by now I hope also to you, is $\left\{(1-x) \mathrm{C}_{3} \mathrm{H}_{8}+x \mathrm{H}_{2} \mathrm{O}\right\}$ for which two widely separated parts of the diagram have been studied: the "gas-gas equilibrium" at $T \approx\left\{\mathrm{T}_{\mathrm{c}}\left(\mathrm{H}_{2} \mathrm{O}\right)=647.1 \mathrm{~K}\right\}$, shown to be of the "second kind" (q.v.), and the tiny region, described by Dr Ewing (21) yesterday, between $T \mathrm{C}\left(\mathrm{C}_{3} \mathrm{H}_{8}\right)=369.82 \mathrm{~K}$ and the UCEP at $T^{\mathrm{U}}=369.52 \mathrm{~K}$.

A good deal of attention has been paid (e.g. Refs. 4, 7, 22) to the taxonomy of phase diagrams that include a $3 \mathrm{~F}$ region, each scheme illustrating some supposed "evolution" from one type of diagram into another. None of these schemes is completely satisfying. I greatly dislike diagrams drawn freehand to describe "general" types of behaviour, preferring those plotted one at a time each for a real mixture. Nevertheless, Fig. 13 is just such a drawing!
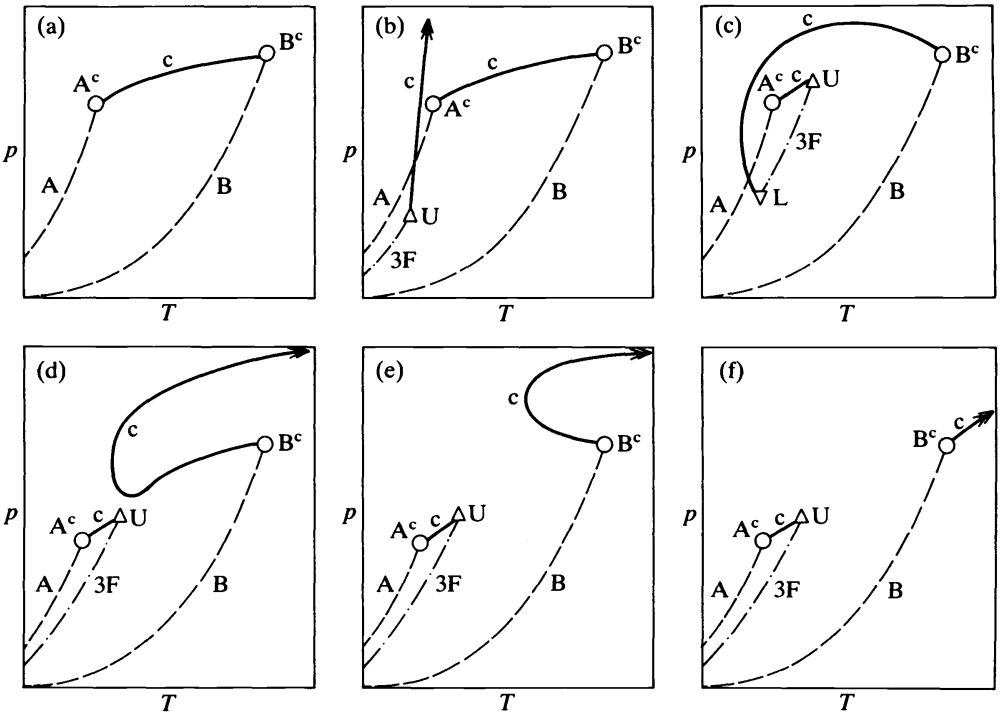

Fig. 13. Freehand sketches of an attempted classification of phase diagrams showing, except in (a), three fluid phases. 
In (a) there is no $3 F$ region. In (b) the $3 F$ line terminates in a UCEP from which a critical line "goes on for ever", the critical points of A and B being joined as in (a). In (c) the critical line starting from the critical point $\mathrm{B}^{\mathrm{C}}$ of $\mathrm{B}$ goes to a lower critical end point ("LCEP"), from which a three-phase line $3 F$ goes to a UCEP, from which a critical line connects to the critical point $A^{C}$ of $A$. In (d) the critical line from $B^{c}$ starts as though it will do the same as in (c) but then "changes its mind", passes through a minimum in $p$ and then through a minimum in $T$, and eventually, "going on for ever", reaches temperatures greater than $T_{\mathrm{B}}^{\mathrm{C}}$, at which we can, if we wish, describe its behaviour as "gas-gas immiscibility". (We must recognize, however, that the densities are typical of "liquids", that no distinction exists in the phase behaviour on one or the other side of $T$, and that "gas-gas immiscibility" so defined would include also phase equilibria at temperatures greater than $T_{\AA}^{C}$ or $T_{\mathrm{B}}^{\mathrm{C}}$ in any example of negative azeotropy.) The UCEP is again joined to the critical point $A^{c}$ of $A$. In (e) the behaviour is similar, except that the critical curve that starts from $\mathrm{B}^{\mathrm{C}}$ does not pass through a minimum in $p$; this, and the behaviour in (d), are called "gas-gas immiscibility of the second kind". In ( $f$ ) the critical curve that starts from $\mathrm{B}^{\mathrm{C}}$ proceeds at once with $\mathrm{dp} / \mathrm{d} T>0$ into the region of "gas-gas immiscibility"; this case is described as being "of the first kind". Whereas $\left\{(1-x) \mathrm{CO}_{2}+x \mathrm{CH}_{4}\right\}$ behaves like (a), $\left\{(1-x) \mathrm{CO}_{2}+x \mathrm{C}_{8} \mathrm{H}_{18}\right\}$ behaves like (b) and $\left\{(1-x) \mathrm{CO}_{2}+x_{16} \mathrm{H}_{34}\right\}$ like (d). $\left\{(1-x) \mathrm{CO}_{2}+x \mathrm{C}_{6} \mathrm{H}_{5} \mathrm{NO}_{2}\right\}$ behaves like (c), $\left.\{1-x) \mathrm{CO}_{2}+x \mathrm{H}_{2} \mathrm{O}\right\}$ like (e), and $\left\{(1-x) \mathrm{He}^{2}+\mathrm{CO}_{2}\right\}$ like (f). $\left\{(1-x) \mathrm{CH}_{4}+x \mathrm{C}_{6} \mathrm{H}_{14}\right\}$ behaves like (c) while $\left\{(1-x) \mathrm{CH}_{4}+x c-\mathrm{C}_{5} \mathrm{H}_{10}\right\}$ behaves like (b) and $\left\{(1-x) \mathrm{CH}_{4}+x c-\mathrm{C}_{5} \mathrm{H}_{9} \mathrm{CH}_{3}\right\}$ like (d), while $\left\{(1-x) \mathrm{He}+x \mathrm{CH}_{4}\right\}$ behaves like $(f) . \quad\left\{(1-x) \mathrm{C}_{4} \mathrm{H}_{10}+x \mathrm{H}_{2} \mathrm{O}\right\}$ behaves like (e) while $\left\{(1-x) \mathrm{C}_{6} \mathrm{H}_{6}+x \mathrm{H}_{2} \mathrm{O}\right\}$ behaves like (d) and $\left\{(1-x) \mathrm{C}_{6} \mathrm{H}_{5} \mathrm{C}_{6} \mathrm{H}_{5}+x \mathrm{H}_{2} \mathrm{O}\right\}$ like (b).

Figure 14 shows diagrams for $\left\{(1-x) \mathrm{CF}_{4}+{ }_{\mathrm{c}} \mathrm{CHF}_{3}\right\}$, like Fig. 13(b), in the neighbourhood of the UCEP. The slope $\partial p c / \partial T$ is drawn much less steeply than
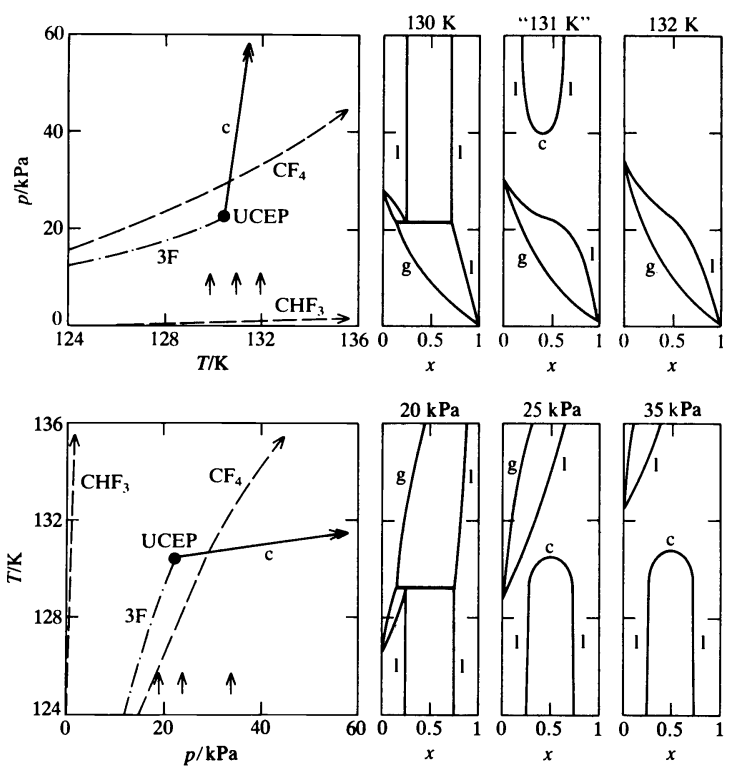

Fig. 14. The $p(T)$ projection and three $p(x)$ sections, and the $T(p)$ projection and three $T(x)$ sections, for

$\left\{(1-x) \mathrm{CF}_{4}+x \mathrm{CHF}_{3}\right\}$, with $\mathrm{d} p / \mathrm{d} T$ drawn less steeply than in reality lest it appear to be vertical.

it is in reality; properly drawn it would appear to be vertical. [We have $\partial p c / \partial T=\left(\partial^{2} S_{\mathrm{m}}^{\mathrm{c}} / \partial^{2}\right) /\left(\partial^{2} V_{\mathrm{m}}^{\mathrm{c}} / \partial x^{2}\right)$. At a UCST $\partial^{2} S_{\mathrm{m}}^{\mathrm{c}} / \partial x^{2}<0$. Thus $\partial p^{\mathrm{c}} / \partial T$ takes its sign from $\partial^{2} V_{\mathrm{m}}^{\mathrm{c}} / \partial x^{2}$ which is usually negative so that $\partial p^{c} / \partial T$ is usually positive.] If $\partial p^{\mathrm{c}} / \partial T$ were negative then the 11 isothermal section at $130 \mathrm{~K}$ would be truncated at an upper critical solution pressure. 
Figure 15 shows the diagram for $\left\{(1-x) \mathrm{CH}_{4}+x \mathrm{C}_{6} \mathrm{H}_{14}\right\}$, pieced together as

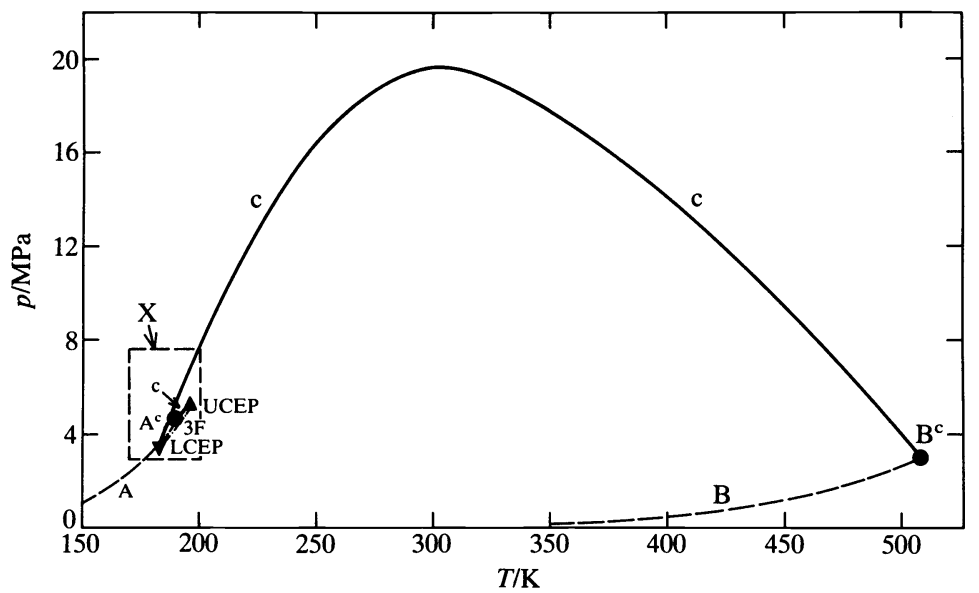

Fig. 15. The $p(T)$ projection for $\left\{(1-x) \mathrm{CH}_{4}+x \mathrm{C}_{6} \mathrm{H}_{14}\right\}$.

best it could be, and Fig. 16 shows the part marked $X$ drawn on a much larger scale.
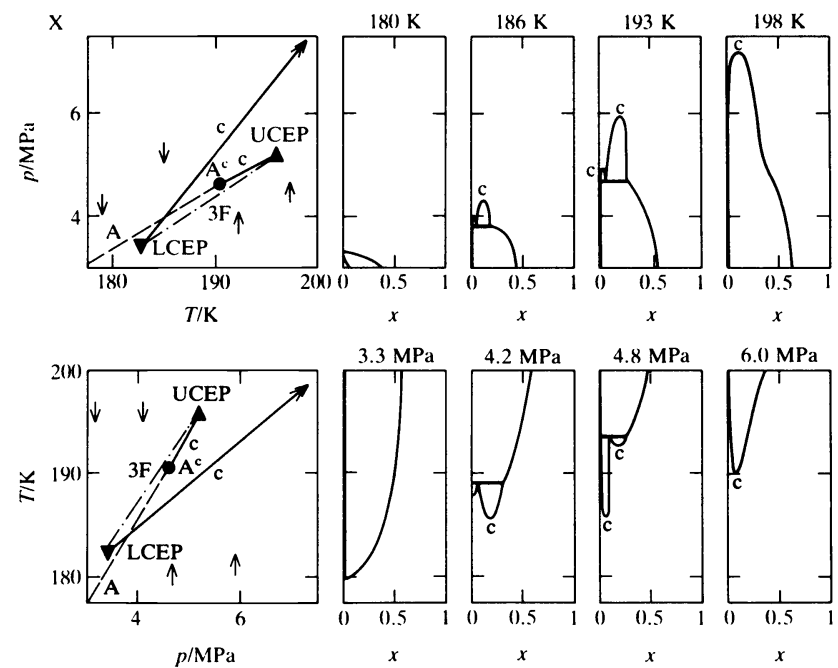

Fig. 16. The $p(T)$ projection and four $p(x)$ sections, and the $T(p)$ projection and four $T(x)$ sections, for the small part marked "X" of Fig. 15 .

In Fig. 17 and Fig. 18, I have again broken my own rule by drawing four examples of different geometrical relations between a critical point $A^{C}$ and an upper critical end point $U$, all designed further to sub-classify Fig. 13 (d) to $(f)$. In Fig. 17, the two examples for $p \AA>p$, lead to distinguishable $p(x)$ sections. In Fig. 18, the two examples for $p_{\mathrm{A}}^{\mathrm{C}}<p^{\mathrm{U}}$ lead to quite different, but again distinguishable, $p(x)$ sections, called in distinction to those of Fig. 17 examples of heteroazeotropy; $\left\{(1-x) \mathrm{C}_{3} \mathrm{H}_{8}+x \mathrm{H}_{2} \mathrm{O}\right\}$ behaves like "3", while $\left\{(1-x) \mathrm{CH}_{3} \mathrm{CH}: \mathrm{CH}_{2}+x \mathrm{H}_{2} \mathrm{O}\right\}$ behaves like " 4 ". 

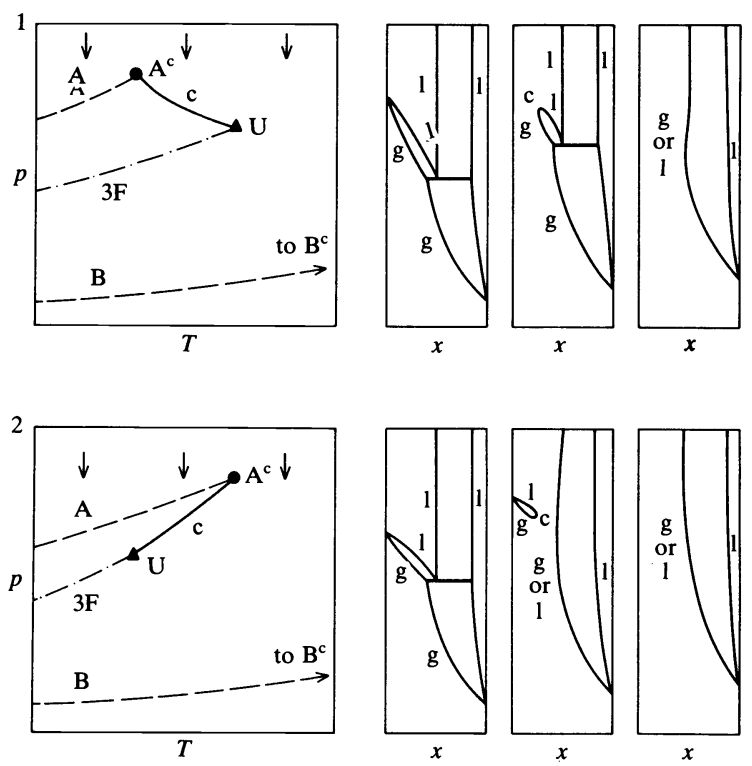

Fig. 17. Freehand sketches of $p(T)$ projections and $p(x)$ sections for three-phase equilibria of the general type of Fig. 13 (d) to (f) for $p \AA>p U: 1$, when $T_{A}^{c}<T^{U}$; and 2 , when $T_{A}^{c}>T^{*}$.
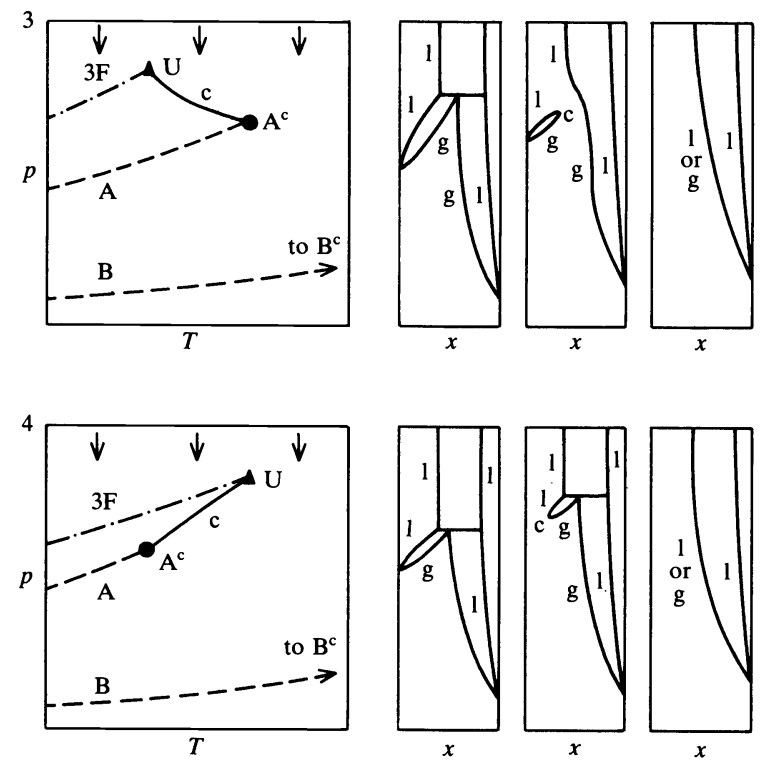

Fig. 18. Freehand sketches of $p(T)$ projections and $p(x)$ sections for three-phase equilibria of the general type of Fig 13 (d) to (f) for $p_{A}^{c}<p{ }_{T}^{U}$ leading to heteroazeotropy: 3 , when $T_{A}^{c}<T^{U}$, and 4 , when ${ }_{T}^{\AA}>T^{U}$.

\section{GAS-GAS IMMISCIBILITY}

Figure 19 shows in its upper part an experimental $p(T, x)$ phase diagram for $\left\{(1-x) \mathrm{He}+x \mathrm{CH}_{4}\right\}$ (Ref. 23), an example of "gas-gas immiscibility of the 

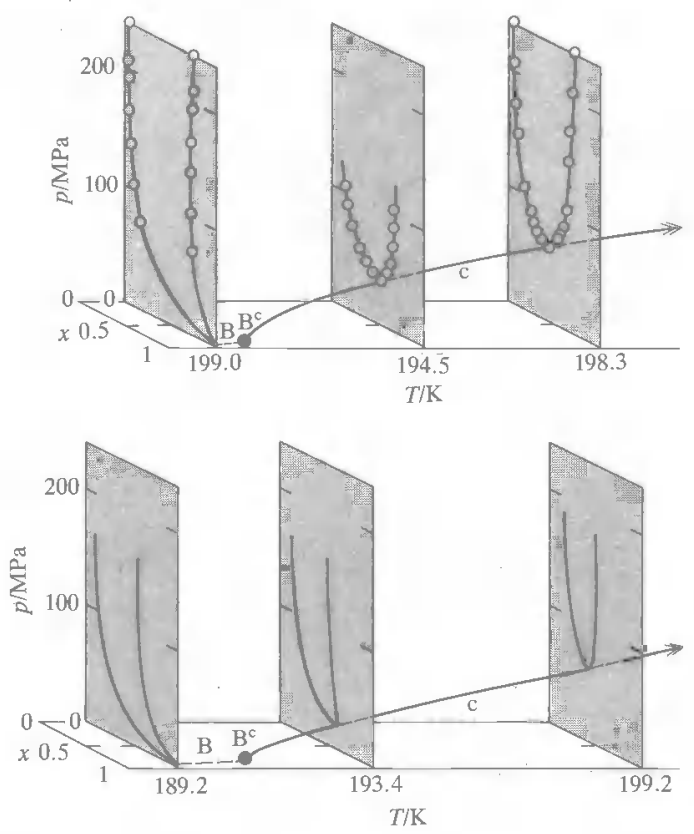

Fig. 19. A "three-dimensional" diagram of "gas-gas immiscibility of the first kind" for $\left\{(1-x) \mathrm{He}+x \mathrm{CH}_{4}\right\}$ with experimental measurements plotted in the upper part and theoretically calculated curves in the lower part.

first kind"; Fig. 20 shows in its upper part an experimental $p(T, x)$ phase diagram for $\{(1-x) \mathrm{He}+x$ Ar $\}$ (Ref. 24), an example of "gas-gas immiscibility

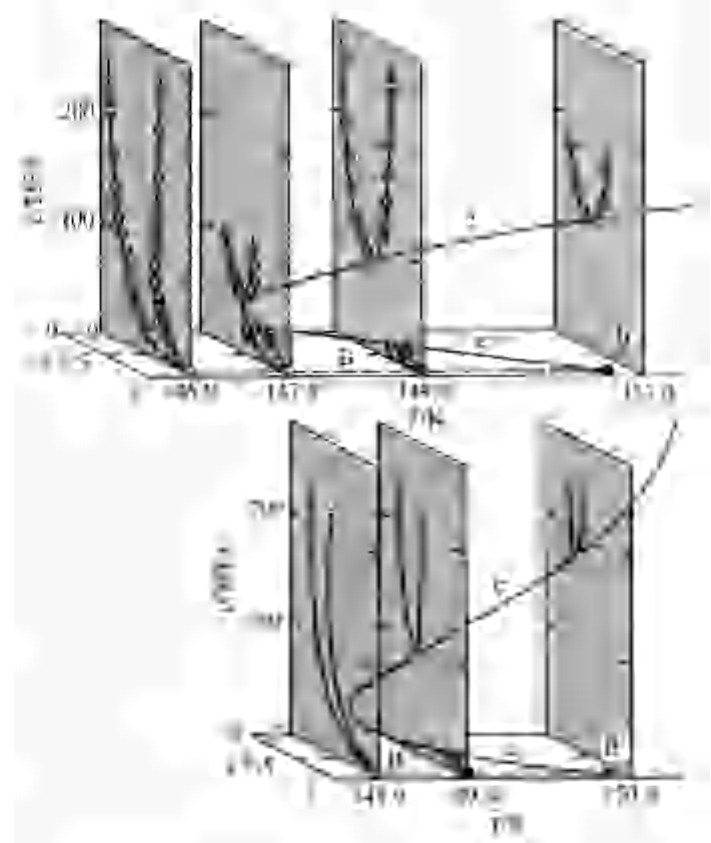

Fig. 20. A "three-dimensional" diagram of "gas-gas immiscibility of the second kind" for $\{(1-x) \mathrm{He}+x \mathrm{Ar}\}$ with experimental measurements plotted in the upper part and theoretically calculated curves in the lower part. 
of the second kind". We see how, for either kind, unmixing persists at temperatures indefinitely far above the critical temperature of methane or of argon and increases with increasing pressure even at enormous pressures.

\section{THEORETICAL CALCULATION OF PHASE DIAGRAMS}

Figure 19 contains in its lower part curves calculated (Ref. 25) from the "one-fluid" theory with Carnahan and Starling's equation of state:

$$
p=R T / V_{\mathrm{m}}+4 b R T /\left(V_{\mathrm{m}}-b\right)^{2}+2 b^{2} R T /\left(V_{\mathrm{m}}-b\right)^{3}-a / V_{\mathrm{m}}^{2} \text {, }
$$

with van der Waal's's prescription for $a(x)$ and $b(x)$ :

$$
\begin{aligned}
& a=(1-x)^{2} a_{\mathrm{AA}}+2 x(1-x) a_{\mathrm{AB}}+x^{2} a_{\mathrm{BB}}, \\
& b=(1-x)^{2} b_{\mathrm{AA}}+2 x(1-x) b_{\mathrm{AB}}+x^{2} b_{\mathrm{BB}},
\end{aligned}
$$

and with the "combining rules":

$$
\begin{aligned}
a_{\mathrm{AB}} & =\xi\left(a_{\mathrm{AA}} a_{\mathrm{BB}} b_{\mathrm{AB}}^{2} / b_{\mathrm{AA}} b_{\mathrm{BB}}\right)^{1 / 2}, \\
b_{\mathrm{AB}} & =(1 / 8)\left(b_{\mathrm{AA}}^{1 / 3}+b_{\mathrm{BB}}^{1 / 3}\right)^{3},
\end{aligned}
$$

having $\xi$ as its only adjustable parameter, chosen for $\left\{(1-x) \mathrm{He}+x \mathrm{CH}_{4}\right\}$ as 0.8 . The values used for the critical temperature and critical pressure of helium were $7.66 \mathrm{~K}$ and $0.551 \mathrm{MPa}$ reckoned (Ref. 26) to be the values that helium would have were its low molar mass not causing quantal deviations from the principle of corresponding states at temperatures as low as its real critical temperature of $5.19 \mathrm{~K}$. Even now such calculations are far from trivial, but it was most satisfying to obtain such good qualitative agreement. Better still, when we used exactly the same equations for $\{(1-x) \mathrm{He}+x \mathrm{Ar}\}$, but now with $\xi=1.02$, we obtained the qualitative agreement shown in the lower part of Fig. 20. That the theory is able not only to predict gas-gas immiscibility but also to distinguish correctly between the first kind for $\left\{(1-x) \mathrm{He}+x \mathrm{CH}_{4}\right\}$ and the second kind for $\{(1-x) \mathrm{He}+x \mathrm{Ar}\}$, the difference arising only from the different critical temperatures and critical pressures of methane (190.6 K, 4.62 MPa) and argon $(150.7 \mathrm{~K}, 4.86 \mathrm{MPa})$, was one of the most satisfying experiences of my scientific life.

Among others who have calculated phase diagrams are van Konynenburg and Scott (22) and Young and his colleagues $(27,28)$, though both groups based their calculations on van der Waals's equation of state. Gubbins and his colleagues (Ref. 29) and others (Ref.3) have thrown new light on the theory of the field by applying perturbation methods to non-spherical molecules to allow for electric dipoles, quadrupoles, and higher multipoles. Another useful theoretical attack is being made by Gibbons and Laughton (30) with a novel version of the Redlich-Kwong equation of state.

\section{Acknowledgement - I am most grateful to Dr Sarah Kimpton for her expert advice.}

\section{REFERENCES}

1. G.M. Schneider, Pure Appl. Chem. 55, 479-492 (1983).

2. J. Zernike, Chemical Phase Theory, Kluwer, Deventer, 1955 , xv + $493 \mathrm{pp}$.

3. J.S. Rowlinson and F.L. Swinton, Liquids and Liquid Mixtures, 3rd Ed., Butterworths, London, 1982 , vii $+328 \mathrm{pp}$.

4. G.M. Schneider in Chemical Thermodynamics, Vol. 2, M.L. McGlashan ed., A Specialist Periodical Report, The Chemical Society, London, 1978, Chap. 4, pp.105-146.

5. C.P. Hicks and C.L. Young, Chem. Rev. 75, 119-175 (1975).

6. M.L. McGlashan, Chemical Thermodynamics, Academic Press, London, 1979, $\mathrm{xix}+345 \mathrm{pp}$., especially Chap.17.

7. I. Wichterle, Fluid Phase Equilibria 1, 161-172 (1977); 1, 225-245 $(1977 / 78) ; 1,305-316(1977 / 78) ; 2,59-78(1978) ; \underline{2}, 143-159$ (1978);

8. M. $\frac{2}{\mathrm{~L}}$. McGlashan and I.R. McKinnon, J. Chem. Thermodynamics $\underline{9}, 1205-1212$ (1977). 
9. D.R. Davies and M.L. McGlashan, J. Chem. Thermodynamics 13, 337-383 (1981).

10. J.A. Hugill and M.L. McGlashan, J. Chem. Thermodynamics 10, 85-93 (1978).

11. M.B. Ewing, R.L. Hurle, and M.L. McGlashan, to be published.

12. W.B. Streett and J.C.G. Calado, J. Chem. Thermodynamics 10, 1089-1100 (1978).

13. G.M. Schneider in Experimental Thermodynamics, Vol.II, Experimental Thermodynamics of Non-reacting Fluids, B. Le Neindre and B. Vodar eds., Butterworths (for IUPAC), London, 1975, Chap.16, pp.787-801.

14. C.L. Young in Chemical Thermodynamics, Vol.2, M.L. McGlashan ed., A Specialist Periodical Report, The Chemical Society, London, 1978, Chap. 3, pp.71-104.

15. J.A. Schouten, A. Deerenberg, and N.J. Trappaniers, Physica 81A, 151-160 (1975).

16. J.A. Hugill and M.L. McGlashan, J. Chem. Thermodynamics 13, 429-436 (1981).

17. W.J. Gaw and F.L. Swinton, Nature 212, 283-284 (1966); Trans. Faraday Soc. $64,2023-2034$ (1968).

18. M.B. Ewing, M.L. McGlashan, and P. Tzias, J. Chem. Thermodynamics $\underline{13}$, 527-535 (1981).

19. M.B. Ewing, Sarah K. Kimpton, and M.L. McGlashan, J. Chem. Thermodynamics $\underline{16}, 669-671$ (1984).

20. Landolt-Börnstein, New Series, IV/3: Thermodynamic Equilibria of Boiling Mixtures, Springer-Verlag, Berlin, 1975, viii + $376 \mathrm{pp}$.

21. M.B. Ewing, S.K. Kimpton, M.L. McGlashan, and J.C. Sanchez, paper \#257 at this Conference.

22. P.H. van Konynenburg and R.L. Scott, Phiz. Trans. Roy. Soc. London A $\underline{298}$, 495-540 (1980).

23. W.B. Streett, A.L. Erickson, and J.L.E. Hill, Phys. Earth Planet. Interiors $\underline{6}, 69-77$ (1972).

24. W.B. Street $\bar{t}$ and J.L.E. Hill, Trans. Faraday Soc. 67, 622-630 (1971).

25. M.L. McGlashan, K. Stead, and C. Warr, J. Chem. Soc. Faraday Trans. II $73,1889-1895$ (1977).

26. E. A. Guggenheim and M.L. McGlashan, Proc. Roy. Soc. London A 206, 448-463 (1951).

27. C.P. Hicks and C.L. Young, J. Chem. Soc. Faraday Trans. II 73, 597-612 (1977).

28. R.L. Hurle, F. Jones, and C.L. Young, J. Chem. Soc. Faraday Trans. II 73, 613-617 (1977).

29. K.E. Gubbins, C.G. Gray, and J.R.S. Machado, Mol. Phys. 42, 817-841, 843-860 (1981), and earlier papers.

30. R.M. Gibbons and A.P. Laughton, J. Chem. Soc. Faraday Trans. II 80, 1019-1038 (1984). 